\title{
Suppression of IRE1 $\alpha$ Attenuated the Fatty Degeneration in Parenteral Nutrition-Related Liver Disease (PNALD) Cell Model
}

\author{
Ningxun Cui, ${ }^{1}$ Mingling Cui, ${ }^{1}$ Jie Li, ${ }^{1}$ Xueping Zhu $\mathbb{D}^{1},{ }^{1}$ and Xiaoli Zhu $\mathbb{D}^{2}$ \\ ${ }^{1}$ Department of Neonatology, Children's Hospital of Soochow University, Suzhou, Jiangsu 215025, China \\ ${ }^{2}$ Department of Intervention, The First Affiliated Hospital of Soochow University, Suzhou, Jiangsu 215006, China
}

Correspondence should be addressed to Xueping Zhu; zhuxueping4637@hotmail.com and Xiaoli Zhu; zhuxiaoli90@hotmail.com Received 16 October 2019; Revised 1 December 2019; Accepted 10 December 2019; Published 3 February 2020

Academic Editor: Luigi Marano

Copyright (C) 2020 Ningxun Cui et al. This is an open access article distributed under the Creative Commons Attribution License, which permits unrestricted use, distribution, and reproduction in any medium, provided the original work is properly cited.

\begin{abstract}
Aims. To model the parenteral nutrition-associated liver disease (PNALD) in rat normal hepatocytes BRL and investigate the role of endoplasmic reticulum stress- (ERS-) related IRE1 $\alpha$ signal in the process of PNALD. Methods. The BRL cells were treated with different concentrations of soybean oil emulsion (SO) to induce hepatocyte fatty degeneration. The PNALD cell disease model was further confirmed by analysis of Oil Red O staining and biochemical parameters. Next, the IRE1 $\alpha$ was silenced by specific shRNAs via lentivirus and the role of IRE $1 \alpha$ in anticytotoxicity and the expression level of ERS-related protein IRE1 $\alpha$ and pIRE1 $\alpha$ were measured. Results. The results of Oil Red O staining indicated that the PNALD was successfully established in BRL cells and the CCK- 8 data indicated which $0.6 \%$ that SO was further applied to the experiment owing to its better induction of PNALD and less toxicity to the cells. Besides, the value of biochemical parameters (TBIL, DBIL, ALT, and AST) was also elevated in the SO group compared with the NG group. After knockdown of IRE1 $\alpha$, the PNALD was also induced while the cells were more tolerant to SO. The less positive Oil Red O staining and reduced values of biochemical parameters were observed in the shIRE1 $\alpha$ group when compared to the shControl, both of which accepted SO treatment. Conclusion. IRE1 $\alpha$ was induced in PNALD cell model and suppression of IRE1 $\alpha$ resulted in reduced steatosis in this cell disease model. Taken together, our data suggested that the IRE1 $\alpha$ pathway may be involved in the development of PNALD.
\end{abstract}

\section{Introduction}

Parenteral nutrition (PN) has revolutionized the lifestyle of the neonates with growth defect caused by intestinal dysfunction [1]. The first case of long-term parenteral nutrition in newborn was reported in the USA last century [2]. Since PN seems to be the best effective therapy on these defects, the number of the patients, both young and aged, depending on PN for survival was growing yearly [3]. Unfortunately, long-term application of PN could develop into serious diseases, such as PN-associated liver disease (PNALD), which could lead to a high incidence of morbidity and mortality [4-6]. According to the report, about $50 \%$ to $66 \%$ of kids receiving long-term PN finally developed into PNALD [7]. Although some recognized risk factors, including premature birth, long-term preservation of PN, low-quality of newborns, and the fat composition [8-10], have been attributed to PNALD, the definitive and specific etiology and pathogenesis still remains uncertain.

In eukaryotic cells, the endoplasmic reticulum (ER) is essential for the folding and trafficking of proteins that enter the secretory pathway. ER orchestrates the synthesis, folding, and transport of at least one-third of the proteins in eukaryotic cells. Because of the high active protein synthetic activity in the hepatocytes, the abundant copy and precise regulation as well as organization of ER were required. Previous studies demonstrated that dysfunction of ER, caused by ER stress, may contribute to human diseases including liver disease $[11,12]$. During the process of ER stress, ER homeostasis will collapse and an unfolded protein response (UPR) get initiated [13]. UPR was regulated by three transducers, inositol-requiring enzyme 1 (IRE1), protein kinase R-like ER kinase (PERK), and activating transcription factor 6 (ATF6), in the ER network [14]. Commonly, they bind to 
glucose-regulated protein 78 (GRP-78) on the ER membrane to promote protein folding and prevent protein aggregation using adenosine triphosphate (ATP). Among the ERresident chaperones, GRP-78 is the master initiator of UPR signaling [15]. Recently, ER stress was reported in the pathogenesis of nonalcoholic fatty liver disease (NAFLD), hepatocellular carcinoma caused by hepatitis B virus, intestinal failure-associated liver disease (IFALD), and alcoholic liver disease (ALD) [16-19]. Zhang et al. reported that ER stress was positively correlated with PNALD and, with activation of autophagy by rapamycin, could protect against PNALD via suppressing ROS-induced ER stress [20]. Our previous study also demonstrated that soybean oil-based lipid emulsions could induce significant ER and mitochondrial damage, ultimately resulting in ER stress in primary rabbit hepatocytes $[21,22]$. Thus, the previous studies suggested that ER stress may be involved in the pathogenesis and development of PNALD.

In this research, rat normal hepatocytes were subjected to soybean oil-based lipid emulsion (SO) treatment to model PNALD. Besides, IRE1 $\alpha$ was suppressed by specific shRNA in hepatocytes to investigate the role of ER stress in PNALD model.

\section{Materials and Methods}

2.1. Rat Normal Hepatocytes. The rat normal hepatocytes (BRL) were kindly provided by Stem Cell Bank, Chinese Academy of Sciences, China. For routine maintenance, the BRL cells were cultured in DMEM (Thermo Fisher, USA) medium containing 10\% FBS (Gibco, US) and 1\% penicillin-streptomycin (Thermo Fisher, USA) at $37^{\circ} \mathrm{C}$ in a $5 \% \mathrm{CO}_{2}$ incubator. The medium was changed daily until required spilt.

2.2. Lentivirus Production. The shRNAs targeting IRE1 $\alpha$ were synthesized by Genewiz and annealed as previously reported. The shRNA product was then ligated into the backbone vector. The virus packaging was processed with psPAX2 (Addgen \#12260) and pMD2.G (Addgen \#12260) into the $293 \mathrm{NT}$ cells. After ultracentrifugation, the virus was collected and resuspended in the medium [23].

2.3. Establishment of IRE1 $\alpha$-Suppressed BRL Cell Lines. According to the IRE1 $\alpha$ protein expression, the shRNA-\#2 was used to transduced the BRL cells. After infection of lentivirus, the cells were selected with puromycin for 3 weeks. Finally, the BRL cells were all GFP positive and the expression of IRE $1 \alpha$ was hardly detected, indicating that the IRE $1 \alpha$-suppressed BRL cell line was successfully established named shIRE1 $\alpha$. Simultaneously, the negative control shRNA was also induced into the BRL cells termed shControl.

2.4. Modeling of PNALD in BRL Cells. According to the previous report [21], the procedure was mildly modified below. The BRL hepatocytes were treated with different concentrations of SO $(0.2 \%, 0.4 \%, 0.6 \%, 1 \%$, and $2 \%$ diluted in DMEM from $20 \% \mathrm{SO}$ ). The $0.6 \%$ concentrations was used for all follow-up experiments. The SO was obtained from SinoSwed Pharmaceutical, China.
2.5. CCK-8 Assay. BRL hepatocytes were seeded into 96well plates at a density of $2 \times 10^{4}$ cells per well and treated as previously described. Cells were then cultured for $24 \mathrm{~h}$ and CCK-8 analysis was performed on them according to the instruction. The absorbance of the formazan derivative was measured at $450 \mathrm{~nm}$ using a microplate reader (DNM-9602; Shengke, Shanghai, China). All measurements were performed in triplicate, and all experiments were repeated three times.

2.6. Oil Red O Staining. The lipid droplet accumulation was detected by Oil Red O staining. Briefly, after cells were treated with SO as indicated, the cells were fixed in $10 \%(v / v)$ formaldehyde for $10 \mathrm{~min}$ and then stained with Oil Red $\mathrm{O}$ solution $(0.5 \%$ in isopropanol, $w / v)$ for $15 \mathrm{~min}$. Following extensive washes with distilled water, cells were stained with hematoxylin for $10 \mathrm{~min}$. The representative images were taken under a light microscope.

2.7. Biochemical Analysis. Culture media from the experimental groups were collected. Various biochemical parameters, including total bilirubin (TBIL), direct bilirubin (DBIL), alanine aminotransferase (ALT), and aspartate aminotransferase (AST), were measured from each sample using an automatic biochemical analyzer (LXTM20, Beckman, USA).

2.8. Western Blotting. The cells were treated as indicated and then washed twice with PBS, followed by lysed in radio immunoprecipitation assay (RIPA) buffer containing $50 \mathrm{mM}$ Tris- $\mathrm{HCl}$ (pH 7.5), $150 \mathrm{mM} \mathrm{NaCl}, 1 \%$ sodium deoxycholate, $1 \%$ Triton X-100, $5 \mathrm{nM}$ ethylenediaminetetraacetic acid, $0.1 \%$ sodium dodecyl sulfate (SDS), and complete proteinase inhibitor (Roche, Mannheim, Germany). After incubation on ice for $30 \mathrm{~min}$, lysates were briefly sonicated and nonsoluble cell debris was removed through centrifugation at $4^{\circ} \mathrm{C}$. Protein lysates in SDS loading buffer were heated for $10 \mathrm{~min}$ at $95^{\circ} \mathrm{C}$ and then electrophoresed in SDS-PAGE gel. Protein samples were transferred onto polyvinylidene fluoride (PVDF) membranes and probed with indicated primary antibodies for $12 \mathrm{~h}$ at $4^{\circ} \mathrm{C}$ after being blocked in $5 \%$ nonfat dry milk. The PVDF membrane was incubated with appropriate secondary antibodies conjugated to horseradish peroxidase for $1 \mathrm{~h}$ at $25^{\circ} \mathrm{C}$. Finally, protein was detected using chemiluminescent horseradish peroxidase substrates with Molecular Imager Gel Doc XR+ System (Bio-Rad).

2.9. Statistical Analysis. All values are expressed as mean \pm SD from replicates, and Student's $t$ test (paired) was performed using GraphPad Prism software. A $P$ value of less than 0.05 was considered statistically significant.

\section{Results}

3.1. Modeling of PNALD in BRL Cells. As presented in Figure 1(a), when the cells treated with SO for $24 \mathrm{~h}$ from $0.1 \%$ to $2 \%$, the extension of Oil Red O staining was increased in a dose-dependent manner, indicating that the lipid droplet accumulation was acquired by administration of SO. Next, to get an optimal SO concentration from 24 to $72 \mathrm{~h}$, cell cytotoxicity was also analyzed by the method of CCK-8. 


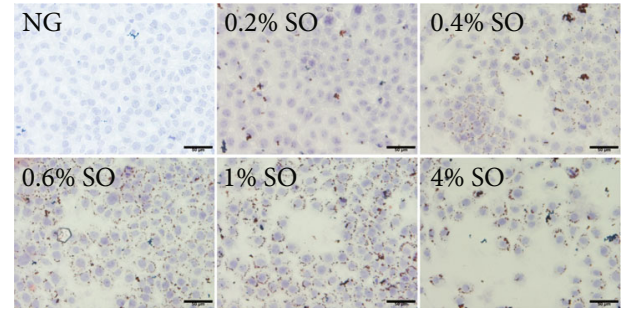

(a)

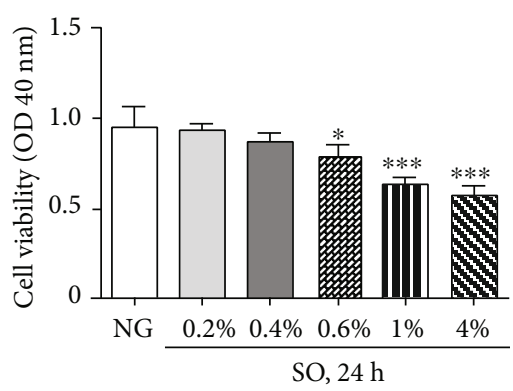

(c)

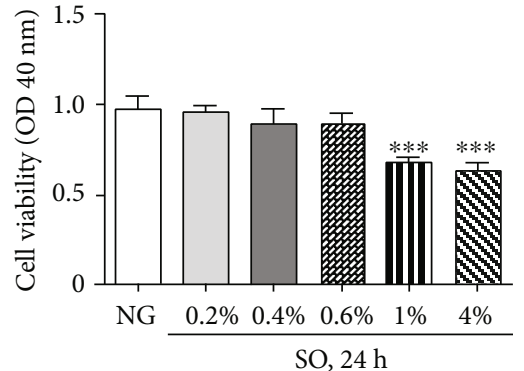

(b)

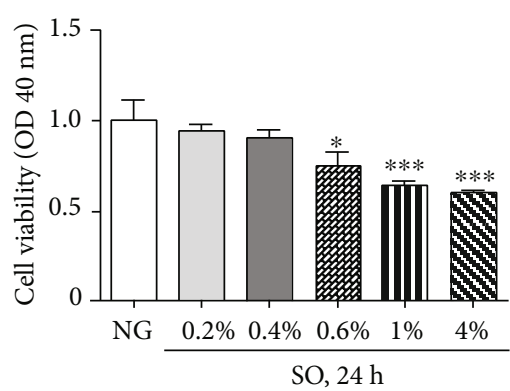

(d)

FIgure 1: Cell modeling of PNALD in vitro. (a) Rat normal hepatocytes BRL were treated with different concentrations of soybean oil emulsion (SO). The degree of steatosis in BRL cells was determined by Oil Red O staining. Scar bar: $50 \mu \mathrm{m}$. (b) BRL cell viability was analyzed by the method of CCK- 8 after treated with SO. NG: not given (BRL cells untreated with SO). The data was presented as mean \pm SD ${ }^{* * *} P<0.001$. (c) BRL cell viability was analyzed by the method of CCK- 8 after treatment with SO for 48 h. NG: not given (BRL cells untreated with SO). The data was presented as mean $\pm \mathrm{SD},{ }^{*} P<0.05,{ }^{* * *} P<0.001$. (d) BRL cell viability was analyzed by the method of CCK-8 after treatment with SO for $72 \mathrm{~h}$. NG: not given (BRL cells untreated with SO). The data was presented as mean \pm SD, ${ }^{*} P<0.05$, ${ }^{* * *} P<0.001$.

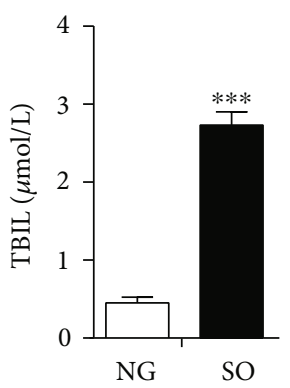

(a)

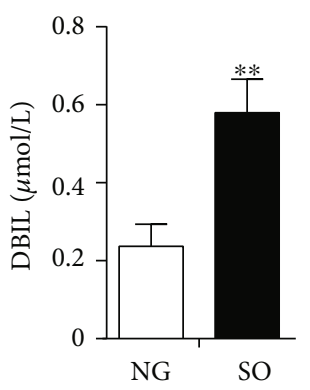

(b)

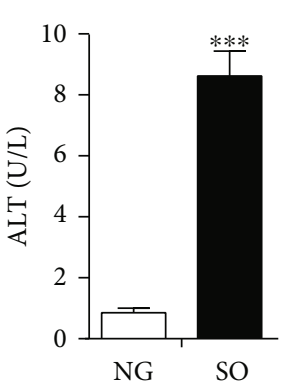

(c)

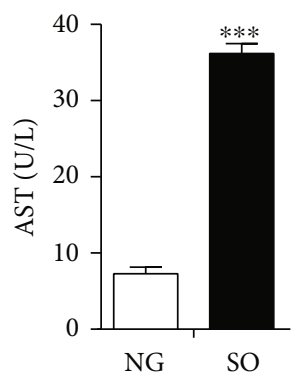

(d)

FIGURE 2: Biochemical parameters of BRL cells after treatment with SO. (a) The content of total bilirubin (TBIL) in cell medium. (b) The content of direct bilirubin (DBIL) in cell medium. (c) The content of alanine aminotransferase (ALT) in cell medium. (d) The content of aspartate aminotransferase (AST) in cell medium. NG: not given (BRL cells untreated with SO). The data was presented as mean \pm SD, ${ }^{* *} P<0.01,{ }^{* * *} P<0.001$.

As shown in Figures 1(b)-1(d), when the cells are treated with SO under the concentration of $0.6 \%$, nonsignificant cytotoxicity was detected, while above this concentration, the BRL cells exhibited serious cytotoxicity. Therefore, $0.6 \%$ SO was applied to further experiments for its greater lipid droplet accumulation and little cytotoxicity.

After treated with SO for $24 \mathrm{~h}$, the function of hepatocytes was analyzed by several biochemical parameters, including TBIL, DBIL, ALT, and AST. When the liver damage occurred, it could result in an accumulation of TBIL, DBIL, ALT, and AST. Surprisingly, all the values of these fac- tors were consistently elevated (Figures 2(a)-2(d)). Taken together, these data suggested $0.6 \%$ SO could induce PANLD in BRL cells, indicating that the BRL-PANLD cell disease model was successfully established.

3.2. Generation of IRE1 $\alpha$-Suppressed BRL Cells. The BRL cells were transduced with lentivirus carrying shControl or shIRE1 $\alpha$. After selection with puromycin $(0.8 \mu \mathrm{g} / \mathrm{mL})$ for 3 weeks, almost all the cells were positive for GFP (Figure 3(a)). The expression of IRE $1 \alpha$ was examined by the method of western blotting, only shIRE1 $\alpha-2 \#$ acquired 


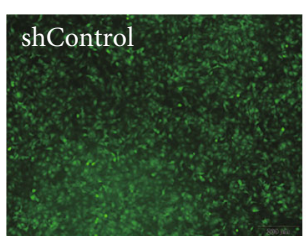

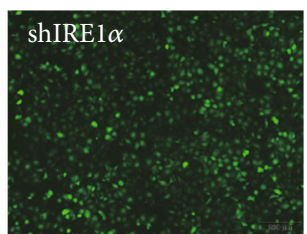

(a)

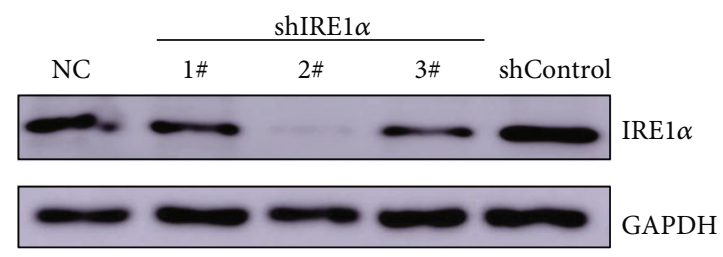

(b)
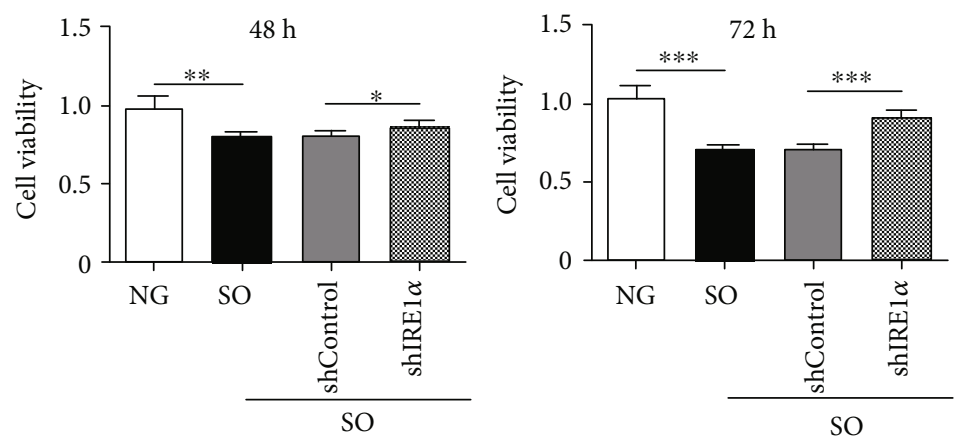

(c)

FIGURE 3: Knockdown of IRE1 $\alpha$ in BRL cells. (a) The representative images of BRL cells transfected with indicated lentivirus. shControl indicated the negative control shRNA and the shIRE1 $\alpha$ was the shRNA targeted shIRE1 $\alpha$. Scar bar: $50 \mu \mathrm{m}$. (b) The protein expression was detected by western blotting. GAPDH was loaded as an internal control. NC indicated BRL cells not infected with any virus. (c) Cell viability was analyzed by the method of CCK- 8 after knockdown of IRE1 $\alpha$ when exposed to SO for $48 \mathrm{~h}$ and $72 \mathrm{~h}$, respectively. NG: not given (BRL cells untreated with $\mathrm{SO}$ ). The data was presented as mean $\pm \mathrm{SD},{ }^{*} P<0.05,{ }^{* *} P<0.01,{ }^{* * *} P<0.001$.

efficient suppression of the target (Figure 3(b)). Thus, the shIRE1 $\alpha$-2\# cell line was subjected to further study. Besides, the SO-induced cytotoxicity was also analyzed after knockdown of IRE $1 \alpha$, and the data obtained from CCK- 8 analysis suggested silence of IRE $1 \alpha$ that could protect cells from cytotoxicity when treated with SO for long term (Figure 3(c)).

\subsection{IRE1 $\alpha$ Suppression Attenuated the Fatty Degeneration in} $B R L$ Cells. Finally, to test whether ER stress-related IRE1 $\alpha$ signal was involved in the development of PANLD in vitro, the cells were treated with $0.6 \% \mathrm{SO}$ as indicated; after staining with Oil Red O, the cell morphology was normal and little lipid droplet accumulation was seen when treated for $0 \mathrm{~h}$. Interestingly, when treated for $24 \mathrm{~h}$, the lipid droplet accumulation was obvious both in the $0.6 \%$ SO group and $0.6 \%$ SO-shControl group, but less lipid droplets are accumulated in the $0.6 \%$ SO-shIRE $1 \alpha$ group, indicating that suppression of IRE1 $\alpha$ could protect against PANLD (Figure 4(a)). Besides, the expression of IRE $1 \alpha$ was also determined after treatment. When treated with $0.6 \% \mathrm{SO}$, the expression of IRE $1 \alpha$ was dramatically increased while little expression was detected in the group of shIRE1 $\alpha$. When ER stress was inhibited by knockdown of IRE $1 \alpha$, the expression of IRE $1 \alpha$ was only accumulated in the cells treated with $\mathrm{SO}$ and SOshControl group (Figure 4(b)). Besides, to further assess the function of hepatocytes, the biochemical parameters, including TBIL, DBIL, ALT, and AST, were analyzed. When treated with SO, compared with the NG group, all the values were increased in the $\mathrm{SO}$ and $\mathrm{SO}$-shControl groups. Once IRE1 $\alpha$ is inhibited although treated with SO, the values of TBIL, DBIL, ALT, and AST were also decreased compared with the shControl group. Taken together, these data suggested that the inhibition of IRE1 $\alpha$ could attenuate the fatty degeneration of PANLD in BRL disease cell model.

\section{Discussion}

In this study, we successfully established rat hepatocytes PNALD cell disease model using soybean oil-based lipid emulsion system. Besides, the changes in several biochemical parameters that reflect hepatic function, which include LD accumulation and ER stress-related signals, were compared between the groups. Furthermore, the IRE1 $\alpha$-suppressed BRL cell line was established to investigate its role in the development of PNALD. The results demonstrated that when IRE $1 \alpha$ is inhibited, the development of PNALD was decreased, suggesting that blocking of ER stress pathway could benefit for liver function when suffering PNALD. Our data suggested that ER stress-related signal was highly activated in PNALD models, which was consistent with the previous reports $[17,24,25]$. The previous clinical trials also demonstrated that the dosage and timespan of lipid emulsion application were positively correlated with the incidence of PNALD [26]. In our data, when treated with high concentration (above $1 \%$ ) of SO for long term ( $48 \mathrm{~h}$ or $72 \mathrm{~h}$ ), the lipid droplet accumulation was much higher than the lower group (under $0.6 \%$ ), this was consistent with the clinical evidence.

The endoplasmic reticulum (ER) governs the proper folding and posttranslational modification of secreted and transmembrane proteins. Diverse physiological and pathological conditions can provoke the accumulation of misfolded proteins within this organelle. These, in turn, can induce ER stress and activate the unfolded protein response (UPR) [27]. Recently, emerging evidence indicates that IRE1 $\alpha$ 

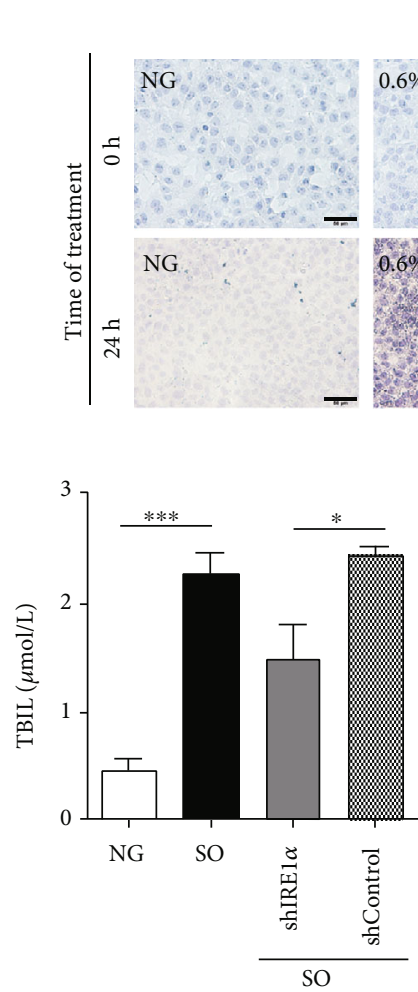

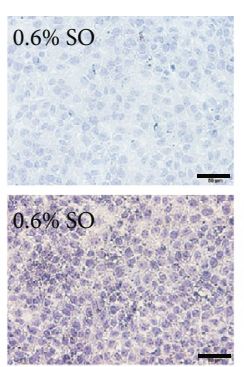

(a)

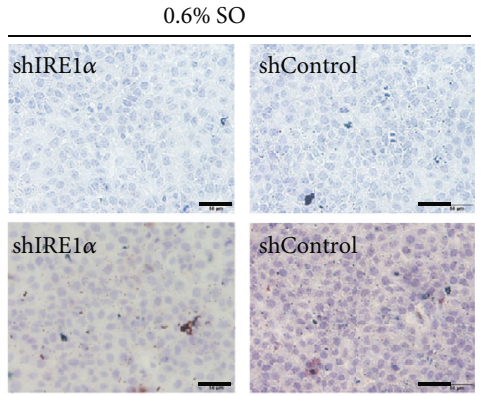

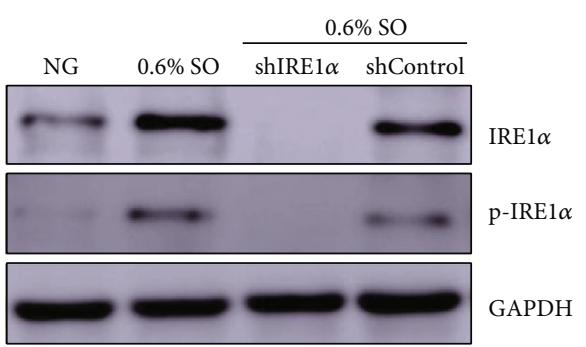

(b)
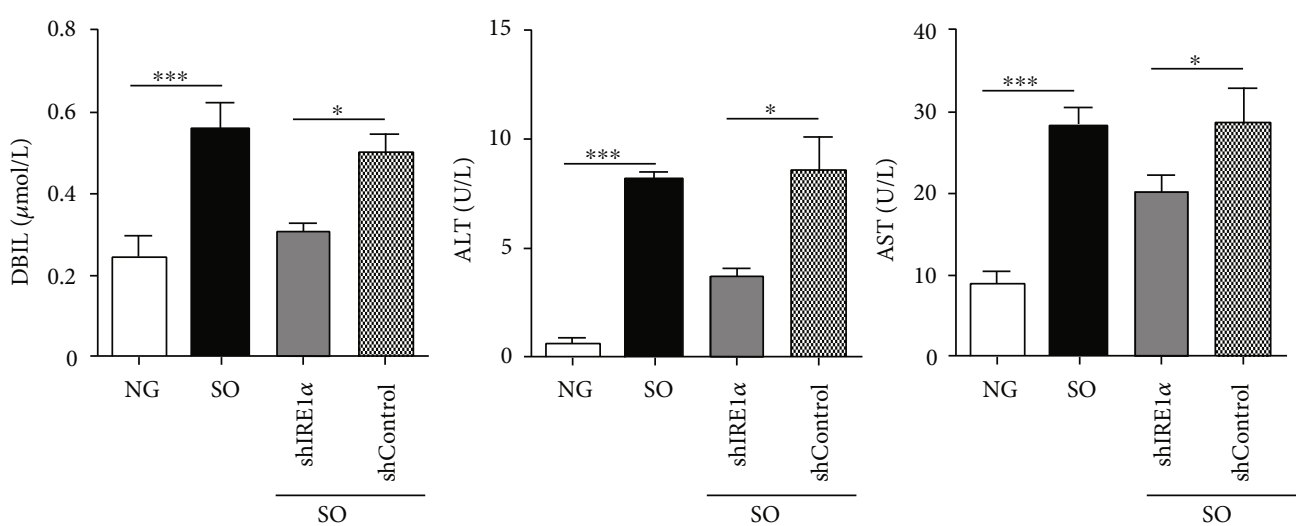

(c)

FIgURE 4: IRE1 $\alpha$ suppression attenuated the steatosis in BRL cells. (a) The representative images of Oil Red O staining in BRL cells in different groups as indicated. Scar bar: $50 \mu \mathrm{m}$. (b) The protein expression was detected by western blotting. GAPDH was loaded as an internal control. NC indicated BRL cells not infected with any virus. (c) Biochemical parameters (TBIL, DBIL, ALT, and AST) of BRL cells in different groups as indicated. NG: not given (BRL cells untreated with SO). The data was presented as mean $\pm \mathrm{SD},{ }^{*} P<0.05,{ }^{* * *} P<0.001$.

signaling can also control UPR-independent cellular pathways, influencing processes such as hepatic lipogenesis, angiogenesis, atherosclerosis, arthritis, and antitumor immunity [28-32]. Whether this conserved signal pathway was involved in the pathogenesis of PNALD was still uncertain. In this study, we first found that IRE1 $\alpha$ was highly expressed in SO-treated BRL cells. When IRE1 $\alpha$ is inhibited, the fatty degeneration was also suppressed. All these data suggested that IRE1 $\alpha$ may be involved in the development of PNALD. But the underlying molecular mechanism still remains to be investigated.

Collectively, soybean oil-based lipid emulsion application could be subjected to induce PNALD disease model in hepatocytes which triggered ER, resulting in lipid droplet accumulation and ER stress. Besides, suppression of IRE1 $\alpha$ attenuated the steatosis in PNALD cells, indicating that IRE $1 \alpha$ pathway may have participated in the development and progression of PNALD.

\section{Data Availability}

All the authors approved and confirmed the data availability, and all the data used to support the findings of this study are included within the article. Besides, the methods and materials are available from the corresponding author upon request.

\section{Disclosure}

Ningxun Cui and Mingling Cui are considered as co-first authors.

\section{Conflicts of Interest}

No conflict of interests existed.

\section{Authors' Contributions}

Ningxun Cui and Mingli Cui performed the experiments, data analysis, and preparation of the manuscript. Jie Li participated in the experiments. Xueping Zhu and Xiaoli Zhu supervised the design and execution of the study andn participated in data analysis, interpretation of data, and revising of the paper. All the authors read and approved the final paper. Ningxun Cui and Mingling Cui contributed equally to this manuscript.

\section{Acknowledgments}

This study was financially supported by the following grants: the National Natural Science Foundation of China (NSFC; Nos. 81771626 and 81971423); the Jiangsu Provincial Maternal and Child Health Key Talents Project (No. FRC201731); the Diagnosis and Treatment Technology Project of Clinical 
Key Diseases in Jiangsu Province (No. LCZX201612); and the Minsheng Technology-Key Technology Application Research Project (No. SS201644).

\section{References}

[1] M. Cahova, M. Bratova, and P. Wohl, "Parenteral nutritionassociated liver disease: the role of the gut microbiota," Nutrients, vol. 9, no. 9, 2017.

[2] S. J. Dudrick, D. W. Wilmore, H. M. Vars, and J. E. Rhoads, "Long-term total parenteral nutrition with growth, development, and positive nitrogen balance," Surgery, vol. 64, no. 1, pp. 134-142, 1968.

[3] S. J. Rangel, C. M. Calkins, R. A. Cowles et al., "Parenteral nutrition-associated cholestasis: an American Pediatric Surgical Association Outcomes and Clinical Trials Committee systematic review," Journal of Pediatric Surgery, vol. 47, no. 1, pp. 225-240, 2012.

[4] T. C. Willis, B. A. Carter, S. P. Rogers, K. M. Hawthorne, P. D. Hicks, and S. A. Abrams, "High rates of mortality and morbidity occur in infants with parenteral nutrition-associated cholestasis," JPEN Journal of Parenteral and Enteral Nutrition, vol. 34, no. 1, pp. 32-37, 2010.

[5] L. Norsa, E. Nicastro, A. di Giorgio, F. Lacaille, and L. D'Antiga, "Prevention and treatment of intestinal failureassociated liver disease in children," Nutrients, vol. 10, no. 6 , p. 664, 2018.

[6] B. Zemrani and J. E. Bines, "Monitoring of long-term parenteral nutrition in children with intestinal failure," JGH Open, vol. 3, no. 2, pp. 163-172, 2019.

[7] J. C. Israelite, "Pediatric parenteral nutrition-associated liver disease," Journal of Infusion Nursing, vol. 40, no. 1, pp. 5154, 2017.

[8] K. Vongbhavit and M. A. Underwood, "Predictive value of the aspartate aminotransferase to platelet ratio index for parenteral nutrition-associated cholestasis in premature infants with intestinal perforation," JPEN Journal of Parenteral and Enteral Nutrition, vol. 42, no. 4, pp. 797-804, 2018.

[9] S. Costa, R. Iannotta, L. Maggio, G. Barone, F. Serrao, and G. Vento, "Fish oil-based lipid emulsion in the treatment of parenteral nutrition-associated cholestasis," Italian Journal of Pediatrics, vol. 44, no. 1, p. 101, 2018.

[10] G. Guthrie, M. Premkumar, and D. G. Burrin, "Emerging clinical benefits of new-generation fat emulsions in preterm neonates," Nutrition in Clinical Practice, vol. 32, no. 3, pp. 326336, 2017.

[11] H. Malhi and R. J. Kaufman, "Endoplasmic reticulum stress in liver disease," Journal of Hepatology, vol. 54, no. 4, pp. 795809, 2011.

[12] F. L. Wu, W. Y. Liu, S. van Poucke et al., “Targeting endoplasmic reticulum stress in liver disease," Expert Review of Gastroenterology \& Hepatology, vol. 10, no. 9, pp. 1041-1052, 2016.

[13] C. Hetz, E. Chevet, and S. A. Oakes, "Proteostasis control by the unfolded protein response," Nature Cell Biology, vol. 17, no. 7, pp. 829-838, 2015.

[14] S. Hummasti and G. S. Hotamisligil, "Endoplasmic reticulum stress and inflammation in obesity and diabetes," Circulation Research, vol. 107, no. 5, pp. 579-591, 2010.

[15] M. Cnop, F. Foufelle, and L. A. Velloso, "Endoplasmic reticulum stress, obesity and diabetes," Trends in Molecular Medicine, vol. 18, no. 1, pp. 59-68, 2012.
[16] N. U. Ashraf and T. A. Sheikh, "Endoplasmic reticulum stress and oxidative stress in the pathogenesis of non-alcoholic fatty liver disease," Free Radical Research, vol. 49, no. 12, pp. 14051418, 2015.

[17] Y. Gu, Y. Yang, X. Cao et al., "Plin3 protects against alcoholic liver injury by facilitating lipid export from the endoplasmic reticulum," Journal of Cellular Biochemistry, vol. 120, no. 9, pp. 16075-16087, 2019.

[18] S. Y. Kim, Y. Y. Kyaw, and J. Cheong, "Functional interaction of endoplasmic reticulum stress and hepatitis B virus in the pathogenesis of liver diseases," World Journal of Gastroenterology, vol. 23, no. 43, pp. 7657-7665, 2017.

[19] L. M. Sharkey, S. E. Davies, A. Kaser, and J. M. Woodward, "Endoplasmic reticulum stress is implicated in intestinal failure-associated liver disease," JPEN Journal of Parenteral and Enteral Nutrition, vol. 40, no. 3, pp. 431-436, 2016.

[20] T. Zhang, J. Yan, N. Wang, L. Dai, Y. Wang, and W. Cai, "Autophagy may protect against parenteral nutritionassociated liver disease by suppressing endoplasmic reticulum stress," JPEN Journal of Parenteral and Enteral Nutrition, vol. 43, no. 1, pp. 96-106, 2019.

[21] X. Zhu, Z. Xiao, Y. Xu et al., "Differential impacts of soybean and fish oils on hepatocyte lipid droplet accumulation and endoplasmic reticulum stress in primary rabbit hepatocytes," Gastroenterology Research and Practice, vol. 2016, Article ID 9717014, 10 pages, 2016.

[22] X. Zhu, X. Zhang, L. Yu, Y. Xu, X. Feng, and J. Wang, "Hepatic Overexpression of GRP94 in a Rabbit Model of Parenteral Nutrition- Associated Liver Disease," Gastroenterology Research and Practice, vol. 2015, Article ID 269831, 8 pages, 2015.

[23] M. J. Benskey and F. P. Manfredsson, "Lentivirus production and purification," Methods in Molecular Biology, vol. 1382, pp. 107-114, 2016.

[24] S. Mohan, M. R. Preetha Rani, L. Brown, P. Ayyappan, and K. G. Raghu, "Endoplasmic reticulum stress: a master regulator of metabolic syndrome," European Journal of Pharmacology, vol. 860, p. 172553, 2019.

[25] S. Torres, A. Baulies, N. Insausti-Urkia et al., "Endoplasmic reticulum stress-induced upregulation of STARD1 promotes acetaminophen-induced acute liver failure," Gastroenterology, vol. 157, no. 2, pp. 552-568, 2019.

[26] V. E. de Meijer, K. M. Gura, H. D. le, J. A. Meisel, and M. Puder, "Fish oil-based lipid emulsions prevent and reverse parenteral nutrition-associated liver disease: the Boston experience," JPEN Journal of Parenteral and Enteral Nutrition, vol. 33, no. 5, pp. 541-547, 2009.

[27] M. Song and J. R. Cubillos-Ruiz, "Endoplasmic reticulum stress responses in Intratumoral immune cells: implications for cancer immunotherapy," Trends in Immunology, vol. 40, no. 2, pp. 128-141, 2019.

[28] Q. Qiu, Z. Zheng, L. Chang et al., “Toll-like receptor-mediated IRE $1 \alpha$ activation as a therapeutic target for inflammatory arthritis," The EMBO Journal, vol. 32, no. 18, pp. 2477-2490, 2013.

[29] O. Tufanli, P. Telkoparan Akillilar, D. Acosta-Alvear et al., "Targeting IRE1 with small molecules counteracts progression of atherosclerosis," Proceedings of the National Academy of Sciences of the United States of America, vol. 114, no. 8, pp. E1395-E1404, 2017.

[30] S. Chopra, P. Giovanelli, P. A. Alvarado-Vazquez et al., "IRE1 $\alpha-$ XBP1 signaling in leukocytes controls prostaglandin 
biosynthesis and pain," Science, vol. 365, no. 6450, p. eaau6499, 2019.

[31] J. R. Cubillos-Ruiz, P. C. Silberman, M. R. Rutkowski et al., "ER stress sensor XBP1 controls anti-tumor immunity by disrupting dendritic cell homeostasis," Cell, vol. 161, no. 7, pp. 15271538, 2015.

[32] Q. Duan, L. Ni, P. Wang et al., "Deregulation of XBP1 expression contributes to myocardial vascular endothelial growth factor-a expression and angiogenesis during cardiac hypertrophyin vivo," Aging Cell, vol. 15, no. 4, pp. 625633, 2016. 